\title{
Evaluation of the oxidase like activity of nanoceria and its application in colorimetric assays
}

\author{
Akhtar Hayat, ${ }^{\text {ab }}$ Jessica Cunningham, ${ }^{\mathrm{a}}$ Gonca Bulbul ${ }^{\mathrm{a}}$ and Silvana Andreescu ${ }^{\mathrm{a}}$ * \\ ${ }^{a}$ Department of Chemistry and Biomolecular Science, Clarkson University, Potsdam, NY, \\ USA. Fax: 3152686610; Tel: 3152682394; \\ ${ }^{b}$ Interdisciplinary Research Centre in Biomedical Materials (IRCBM), COMSATS Institute \\ of Information Technology (CIIT), Lahore, Pakistan
}

*Corresponding author. E-mail:eandrees@clarkson.edu 


\section{Abstract}

Nanomaterial-based enzyme mimics have attracted considerable interest in chemical analysis as alternative catalysts to natural enzymes. However, the conditions in which such particles can replace biological catalysts and their selectivity and reactivity profiles are not well defined. This work explored the oxidase like properties of nanoceria particles in the development of colorimetric assays for the detection of dopamine and catechol. Selectivity of the system with respect to several phenolic compounds, the effect of interferences and real sample analysis are discussed. The conditions of use such as buffer composition, selectivity, $\mathrm{pH}$, reaction time and particle type are defined. Detection limits of 1.5 and $0.2 \mu \mathrm{M}$ were obtained with nanoceria for dopamine and catechol. The same assay could be used as a general sensing platform for the detection of other phenolics. However, the sensitivity of the method varies significantly with the particle type, buffer composition, $\mathrm{pH}$ and with the structure of the phenolic compound. The results demonstrate that nanoceria particles can be used for the development of cost effective and sensitive methods for the detection of these compounds. However, the selection of the particle system and experimental conditions is critical for achieving high sensitivity. Recommendations are provided on the selection of the particle system and reaction conditions to maximize the oxidase like activity of nanoceria.

Keywords: nanoceria particles, oxidase mimic, dopamine, catechol, colorimetric assay 


\section{Introduction}

Natural enzymes have been extensively studied, due to their high enzyme substrate specificity and improved efficiency under mild conditions [1]. They play vital roles in medicine, chemical industry, and agriculture fields. All natural enzymes are proteins except for some catalytic RNA molecules, and are therefore subjected to several intrinsic drawbacks. For example, they can be digested by protease, and can degrade upon exposure to variable environmental conditions. Other disadvantages include a time consuming preparation and purification process, a relatively high cost and the need for specific storage conditions [2]. Therefore, significant interest has been shown for exploration of new and efficient enzyme mimetics. Some level of success has been achieved in this direction with the help of chemically synthesized alternatives [3]. Among enzyme mimetics, non-natural ribosomes and deoxyribosomes have been developed through in vitro selection processes [4]. Other enzyme mimetics including cytochrome P450 mimetic [5], serine proteases mimetics [6], dioxygenase [7], phosphodiesterase [8], ligase [9], nuclease [10] and methanogenesis [11] have been explored.

Recently, inorganic nanomaterials mimicking traditional biological catalysts have received significant interest for their potential application as artificial enzymes $[12,13]$. Their high surface to volume ratio, catalytic activity and abundance of reactive groups on their surface makes these materials powerful candidates as alternatives to biological catalysts. Several types of engineered nanoparticles (NPs) have shown 'enzyme-like' activity, mostly as oxidase, peroxidase and catalase mimetics. some of these have been used as active materials in bioassays, biotechnology and in the biomedical field [12, 14, 15]. NP-based enzyme mimetics offer advantages in terms of cost, stability, ease of production and tenability of catalytic activity. Examples of NP systems with enzyme like activity include: nano-gold [16], 
ferromagnetic materials such as $\mathrm{Fe}_{3} \mathrm{O}_{4}[17], \mathrm{Co}_{3} \mathrm{O}_{4}$ [18], sheet like-FeS nanostructures, $\mathrm{CeO}_{2}$ [19], AgM (silver supported metallic nanoparticles), mesoporous silica encapsulated Pt[13] and metallic NPs supported graphene oxide $[12,20]$. Implementation of these materials in analytical and bioanalytical platforms could rouse development of a new generation of devices with significantly enhanced performances in stability and robustness compared to their enzyme counterparts. However, the conditions in which biomimetic NPs can replace enzymes, their kinetic features and reactivity profiles are highly variable among different types of particles and are generally poorly defined. Whether these NP systems can truly replace natural enzymes and provide the same level of sensitivity and selectivity in analytical assays requires better understanding of their reactivity. It is known that the size, shape, concentration and dispersion medium of the NPs have a significant effect on their reactivity. For example, the peroxidase mimetic activity of rod type $\mathrm{Fe}_{3} \mathrm{O}_{4}$ dominated over the spheres that were much smaller in size [21]. In order for analytical assays based on biomimetic NPs to be developed, it is essential to understand how NP type and environmental conditions affect their reactivity.

In this paper, we provide a comparative investigation of the oxidase mimetic activity of cerium oxide NPs (nanoceria) and define conditions of use (buffer composition, $\mathrm{pH}$ and reaction time) in the design of analytical assays for the detection of phenolic compounds. We compare the kinetic and analytical performance of this particle system versus a model oxidase enzyme, tyrosinase that is commonly used in bioanalytical assays for the detection of these substrates [22-25]. Nanoceria is a redox active inorganic catalyst possessing two oxidation states $\left(\mathrm{Ce}^{+3} / \mathrm{Ce}^{+4}\right)$ and has a highly mobile lattice oxygen located on its surface which facilitates the interchangeable conversion of $\mathrm{Ce}^{+4}$ and $\mathrm{Ce}^{+3}$ [26]. Its unique chemical and electronic configuration confers interesting catalytic and optical properties [27]. These particles have been reported to exhibit catalase and superoxide dismutase like activity [19] 
and have been used for neurodegenerative disease therapy [28-30]. The use of nanoceria particles as a superoxide scavenger [31], protector against photoreceptor degeneration [32], and for the healing of dermal wounds [33] has also been demonstrated. Additionally, they have been used as color indicators and as oxygen sources in oxidase enzyme assays for the detection of glucose [34], dopamine and glutamate [35]. The comparative study reported in this work provides a correlation between the oxidase mimetic activity, the particle size and buffer composition of the NPs that is essential for their future use in analytical assays. It also establishes the optimal $\mathrm{pH}$ range, ionic strength, kinetic parameters and analytical performance metrics of the nanoceria based method.

\section{Experimental section}

\subsection{Chemicals and biochemicals}

Cerium oxide (IV), 20 wt. \% colloidal dispersion in $2.5 \%$ acetic acid, cerium oxide (IV) nano powder; cerium oxide (IV)-20-gadolinium doped nano powder; cerium oxide (IV)-15-samaria doped nano powder and cerium (IV) oxide, nanoparticles, 10 wt. dispersion in $\mathrm{H}_{2} 0$ were from Aldrich. Cerium oxide (IV), 20 wt. \% in water colloidal dispersion was from Alfa Aesar, while $\mathrm{CeO}_{2}$ powder was from Sky spring. Dopamine hydrochloride was obtained from Sigma, while catechol (99\%) was from Acros. Tyrosinase (E.C.1.14.18.1, from mushroom, 4276 units/mg as per supplier information) was prepared in phosphate buffer solution (PBS). Buffer components for PBS including sodium phosphate and potassium phosphate were from ACROS and Sigma-Aldrich, respectively.

\subsection{Solutions and buffer}


The PBS was prepared from $0.1 \mathrm{M}$ sodium phosphate and $0.1 \mathrm{M}$ potassium phosphate in distilled water. The Enzyme stock solution $(1313 \mathrm{mU} / \mathrm{mL})$ was prepared in PBS. Dopamine and catechol were first dissolved in methanol at a concentration of 250 and $19 \mathrm{mM}$, respectively, depending on their solubility in the solvent. Dilutions were prepared from the stock solution in distilled deionized water and PBS of various $\mathrm{pH}$ and ionic strengths, depending upon the nature of experiments. The NP dispersion was diluted in distilled deionized water and PBS, and sonicated for $5 \mathrm{~min}$ prior to all experiments. Distilled, deionized water (Millipore, Direct-Q-system) with a resistivity of $18.2 \Omega \mathrm{cm}$ was used for the preparation of the buffer solutions.

\subsection{Instrumentation}

UV-Vis spectrophotometric measurements were performed with a Schimadzu P2041 spectrophotometer equipped with a 1-cm path length cell. Measurement of zeta potential to evaluate surface charge of the nanoceria particles in various ionic strength solutions was investigated by using a Zeta PALS from Brookhaven Instruments Corporation.

\subsection{Experimental procedures}

To investigate the oxidase like activity of nanoceria, particle dispersions were exposed to varying concentrations of dopamine and catechol. Dopamine and catechol were added to the particle dispersions at concentrations of 0.8 and $0.35 \mathrm{mM}$, respectively. The optical properties of the oxidation products were measured spectrophotometrically. The effect of the type and concentration of particles, the $\mathrm{pH}$ and ionic strength for seven types of commercially available nanoceria were compared to those of a tyrosinase enzyme for these substrates. The effect of nanoceria concentration was investigated in the concentration range of 0.125 to $1 \times 10^{3} \mathrm{mg} / \mathrm{L}$ using $1 \mathrm{mM}$ dopamine and $0.35 \mathrm{mM}$ catechol. The concentration of nanoceria particles used 
in the optimization tests of $\mathrm{pH}$ and ionic strength was $1 \times 10^{3} \mathrm{mg} / \mathrm{L}$ with a concentration of 0.8 $\mathrm{mM}$ dopamine. The effect of $\mathrm{pH}$ was studied in PBS solutions with a $\mathrm{pH}$ range of 6 to 8 . The effect of ionic strength was studied in PBS solutions of ionic strength compositions varying from $0.06 \mathrm{mM}$ to $1 \mathrm{M}$. The change in the surface charge of the nanoceria particles was assessed by DLS and zeta potential measurements. Analytical measurements to determine dopamine and catechol and to establish method performance were performed in the concentration range of 0.4 to $850 \mu \mathrm{M}$ using $1 \times 10^{3} \mathrm{mg} / \mathrm{L}$ nanoceria dispersion. The absorbance was measured at $480 \mathrm{~nm}$, corresponding to the nanoceria generated quinone complex.

Comparative enzymatic experiments were performed using the tyrosinase enzyme. Enzymatic tests to optimize conditions were performed by varying enzyme activity in the range of 0.625 to $15 \mathrm{mU} / \mathrm{mL}$ in $\mathrm{PBS}$ at a $\mathrm{pH}$ of 6.5 in the presence of $1.4 \mathrm{mM}$ dopamine and $0.75 \mathrm{mM}$ catechol. Effect of the $\mathrm{pH}$ was assessed in PBS in the $\mathrm{pH}$ range from 6 to 8 using 1 $\mathrm{mM}$ dopamine and $0.65 \mathrm{mM}$ catechol. Optimal concentrations were determined based on absorbance values corresponding to the enzymatically generated quinones. Detection of dopamine and catechol was performed in the concentration range of 0.075 to $2 \mathrm{mM}$. Substrates were added to tyrosinase in PBS at a pH of 6.5 and the absorbance at $476 \mathrm{~nm}$ for dopamine and at $388 \mathrm{~nm}$ for catechol were measured to determine kinetic and analytical parameters.

\section{Results and Discussion}

\subsection{Oxidase like activity of nanoceria: proof of concept for nanoceria based detection}

The dual $\left(\mathrm{Ce}^{+4} / \mathrm{Ce}^{+3}\right)$ oxidation state and the high mobility of surface oxygen are responsible for the oxidase like activity of nanoceria. To demonstrate this activity, we have selected two 
phenolic compounds, dopamine and catechol, and have studied their catalytic oxidation by nanoceria. Scheme 1 illustrates the oxidation of dopamine and catechol by nanoceria and the corresponding absorption spectra for a20-25 $\mathrm{nm}$ nanoceria dispersion $\left(1 \times 10^{-3} \mathrm{mg} / \mathrm{L}\right)$. The color of the particle dispersion changed instantaneously from transparent/light yellow to brown upon addition of dopamine and catechol. The intensity of the color is dependent on the concentration of dopamine and catechol, and varies with the type of nanoceria particle used. The color change is due to the oxidation of the phenolic compounds by nanoceria and formation of strongly absorbing charge transfer complexes. Oxidation and formation of charge transfer complexes between ceria and dopamine was demonstrated previously with FTIR spectroscopy, XPS and UV-VIS spectroscopy [36]. The extent of change in optical properties of nanoceria particles upon exposure to phenolic compounds is associated to the ratio of $\mathrm{Ce}^{+4}$ and $\mathrm{Ce}^{+3}$ in the nanoparticle system and formation of charge transfer complexes. The resulting change in optical properties of the reaction mixture indicates that these particles can be used as an oxidation catalyst to replace the commonly used tyrosinase enzyme, and as a new colorimetric probe for the quantitative detection of phenolic compounds.
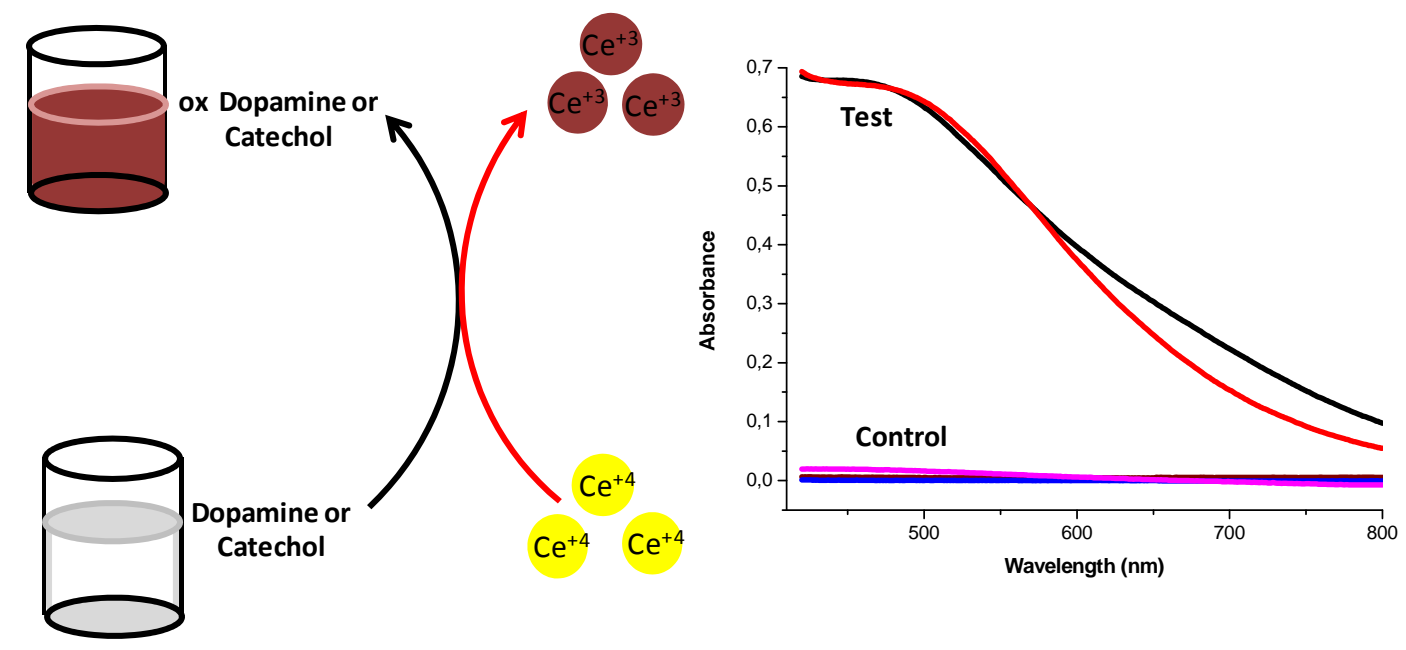

Scheme 1. The principle of nanoceria based detection of dopamine/catechol. Absorption spectra of dopamine and catechol oxidation by nanoceria (Test) and control spectra for the same concentration of dopamine and catechol without particles. 


\subsubsection{Effect of particle type}

It is known that NPs with different physical properties may have different chemical reactivity $[37,38]$. We used changes in the optical properties of nanoceria upon incubation with dopamine and catechol to assess the 'oxidase like activity' of different types of commercially available nanoceria. To demonstrate whether this colorimetric change is a common feature of nanoceria, we tested different types of nanoceria particles from seven different suppliers, both dry powders and colloidal dispersions of pure and doped particles. In addition to pure ceria, sammaria and gandolinium doped ceria were tested because these particles are known to have enhanced oxygen vacancies at their surface and potentially higher oxidation ability. Table S1 in the Supporting information (SI) provides detailed information on the seven different types of commercially available particles used in this study, including: supplier, particle size, particle size distribution as determined from dynamic light scattering (DLS) and surface charge (zeta potential value). Screening tests were performed with a particle concentration of $1 \times 10^{3} \mathrm{mg} / \mathrm{L}$. Figure 1 shows optical images of vials containing nanoceria dispersion of the seven types of particles in the presence of $1 \mathrm{mM}$ dopamine in water. In the absence of dopamine, all particle dispersions are colorless at the concentration tested (control vials are shown in Figure S1 in SI). As a common feature, all particle types showed absorbance changes when exposed to dopamine and catechol, but with varying intensity (Figure S2). This suggests that the oxidase like activity is a common feature of nanoceria regardless of the synthesis procedure, but as expected, this varies with particle size and agglomeration status. These experiments demonstrate the variability of the oxidase like activity of these particles on the surface and physicochemical properties, as discussed below.

\section{Figure 1}


The highest intensity was observed for aqueous dispersions containing particles of $<30 \mathrm{~nm}$, as determined by PSD (particle size distribution)/DLS measurements. The particles dispersed in acetic acid showed higher absorbance values as compared to the other types of particles. By comparison, the nanoceria powders showed significantly lower changes in optical properties. This behavior can be related to the PSD values of the particles, which were higher in the case of nanoceria and doped nanoceria powders e.g. (137-850 $\pm 8-60 \mathrm{~nm})$ as compared to those of nanoceria dispersions (18.9-78.7 $\pm 1.5-5 \mathrm{~nm})$; see Table S1. Similarly, a decreased zeta potential value was observed in the case of nanoceria and doped nanoceria powders, $(-8.49$ $12 \pm 1.5 \mathrm{mV}$ ) indicating that these particles have a higher tendency to aggregate. By comparison, the nanoceria dispersions were characterized by high zeta potential values (25$35 \pm 2 \mathrm{mV}$ ). This explains the high stability of the dispersion because they conserve their surface reactivity at the nano scale. The different sizes and particle types can also have varying ratios of $\mathrm{Ce}^{3+/ 4+}$ on the surface, and can contain surface ligands that may affect reactivity. The maximum change in color was observed for the nanoceria dispersion in acetic acid, which could be attributed to their lower particle diameter $(18.9 \pm 1.5 \mathrm{~nm})$ and the high positive zeta potential $(+35 \pm 2 \mathrm{~nm})$. This indicates good colloidal stability, probably due to the chelating effect of acetate on ceria. This analysis demonstrates that reactivity varies significantly among different types of particles.

\subsubsection{Effect of $\mathbf{p H}$, ionic strength and buffer composition}

Since the catalytic properties of NPs are highly dependent on the $\mathrm{pH}$ of the reaction medium and buffer anions [39], it was important to investigate the effect of these parameters. Figure S3 shows the effect of $\mathrm{pH}$ on dopamine oxidation for a nanoceria dispersion in the $\mathrm{pH}$ range from 6 to 8 in PBS. The pH effect for the six other types of particles is provided in Figure S4 (SI). The highest activity quantified from the optical changes was observed with nanoceria 
dispersions with PSD values lower than $30 \mathrm{~nm}$. For these particles, a significant color change was observed over the entire $\mathrm{pH}$ range tested from 6 to 8 in PBS. The highest intensity was observed at $\mathrm{pH}$ 8. To further determine the effect of ionic strength, we have tested the oxidation behavior of these particles in PBS solutions with a concentration range from 0.00625 to $0.1 \mathrm{M}$ at a $\mathrm{pH}$ of 8 (Figure 2). Control experiments showing spectra of dopamine and catechol (in the absence of particles) and nanoceria particles without phenolics show no absorption wave in the wavelength range tested (400 to $800 \mathrm{~nm}$ ). This indicates that neither dopamine, nor catechol or nanoceria absorb in that range (Figure S5, SI). The maximum absorption was achieved with phosphate concentrations lower than $0.05 \mathrm{M}$. Higher concentrations induced significant particle aggregation and increased the baseline, and therefore were not used in the assay. The absorbance decreases with the increase in ionic strength in the concentration range tested. This demonstrates that the presence of phosphate anions has a damaging effect on the oxidase activity of these particles. This is evident from the comparison of the absorption spectra of nanoceria in the presence of dopamine in water and in PBS, showing significantly higher absorption characteristics in water. These results indicate that water is a better solvent for achieving enhanced oxidase ability of nanoceria particles.

\section{Figure 2}

The reduced reactivity in the buffer can be attributed to the formation of cerium phosphate on the particle surface [40], resulting in a partial inactivation of the surface. This prevents the reaction with the phenolic compounds by blocking the interconversion between $\mathrm{Ce}^{4+} / \mathrm{Ce}^{3+}$. In order to confirm this hypothesis, DLS and zeta potential experiments were performed to analyze the formation of cerium phosphate and its effect on the aggregation behavior of nanoceria particles $\left(1 \times 10^{3} \mathrm{mg} / \mathrm{L}\right)$. An increase in particle size from $70 \pm 5 \mathrm{~nm}$ to $1154.87 \pm 50$ $\mathrm{nm}$, and change of the zeta potential values from $33 \pm 2 \mathrm{mV}$ to $-50.27 \pm 5$ was observed with 
increasing concentration of PBS $(0.00625-0.1 \mathrm{M})$. The change in the zeta potential value clearly indicates a change in the surface properties of these particles and can be indicative of formation of cerium phosphate. The detailed DLS/zeta potential results are provided in the supplementary Table S2 (SI).

To assess the effect of other anions, dopamine reactivity tests were also performed with citrate and acetate buffers under a varying $\mathrm{pH}$. Nanoceria reactivity was observed to decrease with decreasing $\mathrm{pH}$ for both types of buffers (Figure S6-Figure S7). As compared to phosphate, the color intensity in acetate and citrate was more strongly dependent on the $\mathrm{pH}$. The acetate was more reactive against dopamine than citrate and both showed lower reactivity at lower $\mathrm{pH}$ values. We note, however, that the reactivity in buffers is lower than that measured for the same particle type and dopamine concentration in water. This indicates the high variability of this reaction on the physicochemical properties of these particles as well as on the reaction conditions and buffer environment.

\subsubsection{Effect of particle concentration}

The oxidase like activity is strongly dependent on the available reactive surface of the NPs. To determine the effect of nanoceria concentration, fixed concentrations of dopamine and catechol were incubated with varying concentrations of nanoceria particles ranging from 0.125 to $1 \times 10^{3} \mathrm{mg} / \mathrm{L}$. Figure 3 shows the absorption spectra of different concentrations of water dispersed nanoceria in the presence of dopamine and catechol. It can be seen that the absorption peak increases with increasing the particle concentration for both of the analytes. However, the color intensity for the same particle concentration is different when the same concentration of catechol and dopamine was used. This can be due to the different chemical structure and redox potential which favors catechol oxidation. It should be noted, however, that while higher particle concentrations may lead to an elevated signal, this can also result in 
increased background absorption and light scattering. A concentration of $1 \times 10^{3} \mathrm{mg} / \mathrm{L}$ was selected to prevent aggregation and light scattering for the analytical characterization of the method.

\section{Figure 3}

\subsection{Kinetic analysis and comparison with the enzyme based reaction}

The oxidation of dopamine and catechol by nanoceria is initiated almost instantaneously and can be seen by the immediate appearance of the brown color and the strong absorbance band. A similar behavior for varying concentrations of particles further confirmed the fast reactivity of nanoceria for catechol and dopamine oxidation (Figure S8). The reaction rate for an incubation period of $1 \mathrm{~h}$ is shown in Figure S9. The oxidase like activity was fitted by the classical Michaelis-Menten model (Figure 4). A plot of $\mathrm{V}$ against [S] was used to determine the kinetic parameters. The Km values derived from the slope and intercept of the line were $0.25 \mu \mathrm{M}$ for dopamine and $180 \mu \mathrm{M}$ for catechol. By comparison, the $\mathrm{Km}$ values of the optimized enzymatic reaction using tyrosinase in the same reaction conditions were $0.3 \mu \mathrm{M}$ and $200 \mu \mathrm{M}$ for dopamine and catechol, respectively. Optimization and characterization of the tyrosinase catalyzed enzyme assay and the corresponding absorption spectra and kinetic analysis are summarized in the SI section (Figures S10 to S13).

Figure 4.

\subsection{Nanoceria based detection of dopamine and catechol}

The rapid color change and the strong absorbance characteristics of the oxidation of dopamine and catechol by nanoceria indicate that these particles can be used for oxidation and as color indicator reagents for the detection of these compounds. Characterization of the analytical assay was performed in optimized conditions with water as the solvent, with the aqueous 
nanoceria dispersion in acetic acid and with a concentration of particles of $1 \times 10^{3} \mathrm{mg} / \mathrm{L}$.

Figure 5 displays the calibration curves for dopamine and catechol detection by nanoceria.

The spectroscopic response increases linearly with the increasing concentration of dopamine and catechol, with a linearity range between 1 and $400 \mu \mathrm{M}$ for catechol and between 1 and $800 \mu \mathrm{M}$ for dopamine. The limits of detection (LOD) for dopamine and catechol were 1.5 and $0.2 \mu \mathrm{M}$, respectively, according to the $3 \sigma$ criteria. The graphs in Figure 5 show replicate analysis from three independent trials. This method showed excellent reproducibility with a coefficient of variability of less than 1\% (see Table 2 for a comparative analysis). By comparison, the LODs for dopamine and catechol for the corresponding enzymatic reaction were 50 and $30 \mu \mathrm{M}$. This shows that the nanoceria detection is several times more sensitive than the conventional tyrosinase assay (in optimized conditions). The calibration curves of the enzyme assay for dopamine and catechol are shown in Figure S14.

\section{Figure 5.}

\subsection{Interferences study and real sample analysis}

To investigate the specificity and selectivity of the proposed assay, the reactivity of possible interfering compounds including glucose, glycine, histidine, L-glutamic acid, phenylalanine, $\mathrm{K}$ and $\mathrm{Na}$ ions were tested under similar experimental conditions as those described for dopamine and catechol. Changes in the optical properties of the reaction mixture and UV-Vis study were performed to characterize the reaction. As seen in Figure 6A, these compounds did not show any reaction with the nanoceria particles, and no absorption peaks were observed for these compounds. We further studied the selectivity of the nanoceria assay against different types of phenolic compounds. Figure 6B shows the changes in optical properties and absorption spectra obtained with phenolic compounds. A variation in the nanoceria reactivity was observed depending on the type of phenolic compound tested. The 
highest intensity was obtained for catechol and dopamine while the other phenolics tested do not show immediate color changes. The reaction is dependent on the chemical structure of these compounds and is favored for o-substituted phenols like dopamine and catechol. While the other phenolics tested (quercetin, hydroquinone, bisphenol A and even phenol) do react with nanoceria, the oxidation is slower than that of dopamine and catechol; the sensitivity of the assay for these compounds is limited (a color change is observed for higher concentrations and longer incubation times). We should state that tyrosinase is a general oxidative enzyme for phenolic compounds [25, 41-43] and has limited specificity. In this respect, the results suggest that the nanoceria assay is more selective as compared to tyrosinase detection methods.

The method was further applied to determine presence of dopamine and catechol in real samples. Human serum samples were spiked with varying concentrations of dopamine, while tap water was spiked to detect catechol. No significant difference in spiked and obtained values was observed (Table. 1). The precision of the assay was evaluated by the relative standard deviation $(\% \mathrm{RSD})$ for triplicate measurements. The accuracy $(\% \mathrm{RE})$ of analyte determination was examined by comparing the real and measured values. The change in the optical properties of the reaction mixture and their respective UV-Vis spectra are provided in Figure S15. The results show a good agreement between the spiked and determined concentration with \% Recovery between 95 and $101.6 \%$ for the three concentrations tested. This demonstrates the suitability and potential of the method for real sample analysis.

\subsection{Comparison between the nanoceria and tyrosinase assays}


One of the objectives of this work was to comparatively establish the characteristics of this method versus that of an oxidative enzyme that is commonly used for the detection of phenolics. We have selected for this purpose tyrosinase, a copper protein that is widely used in bioanalytical assays for the detection of phenolic compounds, including dopamine and catechol. We first performed kinetic analysis and optimization tests for the enzyme with the two substrates. The results are included in the SI (Figure S10-S13). The oxidation products of the enzymatic reaction, dopaquinone and quinone show characteristic peaks at $476 \mathrm{~nm}$ and $388 \mathrm{~nm}$, respectively. By comparision, the peaks of the oxidation products by nanoceria are red shifted to 520 and $500 \mathrm{~nm}$ for dopamine and catechol, respectively. We attribute the enhanced spectral features to formation of charge transfer complexes, which could provide increased detection characterisitcs and higher sensitivity for the nanoceria assay.

Table 2 provides a comparison of the analytical performance of the nanoceria and the enzyme based detection of dopamine and catechol. The analytical parameters obtained from kinetic data and fitted calibration curves were used to compare the enzymatic and nanoceria based assays. The comparison of $\mathrm{Km}$ values demonstrates a fast reaction rate and high affinity of the two substrates for the inorganic material. The nanoceria assay exhibits low limits of detection for the two substrates. The sensitivity of the method was 3 folds higher than that of the enzyme assay for the optimized amounts of enzyme and particles that were used. Based on the absorbance values, we estimate that $1 \mathrm{mU} / \mathrm{mL}$ tyrosinase corresponds to $28.3 \mathrm{mg} / \mathrm{ml}$ nanoceria. Tyrosinase oxidation of dopamine and catechol requires a specific $\mathrm{pH}$ medium, while nanoceria particles show maximum reactivity in deionized distilled water. Tyrosinase is a more expensive catalyst, while nanoceria particles can be purchased relatively inexpensively or can be synthesized in the laboratory in large quantities. Moreover, the particles are stable at room temperature and conserve their 'oxidase activity' for years. This is a significant advancement over the enzyme catalyst that is sensitive to changes in temperature and $\mathrm{pH}$, and 
can lose its activity overtime and requires refrigeration. All of these merits make nanoceria particles promising candidates for the development of analytical assays for the detection of phenolic compounds.

\section{Conclusion}

In summary, this work explored the oxidase like properties of nanoceria particles and established the optimum operational parameters and reaction conditions in the development of colorimetric assays for the detection of phenolic substrates. Several types of particles and reaction conditions were tested to establish conditions of use that maximize the oxidase like activity. All particle types showed absorbance changes when exposed to dopamine and catechol in water, but with varying intensity. This suggests that the oxidase like activity is a common feature for nanoceria regardless of the synthesis procedure, but this varies with particle size and agglomeration status. The use of phosphate buffer was found to reduce assay sensitivity due to formation of cerium phosphate on the particle surface. The comparative study with the enzyme counterpart indicated that the nanoceria assay is more sensitive than the tyrosinase assay for detection of dopamine and catechol. The same assay could be used as a general sensing platform for the detection of other phenolics. However, the sensitivity of the method varies significantly with the structure of the phenolic compound and seems to be favored for o-substituted hydroxylated phenols like dopamine and catechol. Further work is needed to establish the structural determinants of the oxidase activity for a larger class of phenolics.

Based on this study, the following general recommendations are provided when considering nanoceria as an oxidation catalyst for the development of analytical assays: (1) the use of water as solvent, (2) the use of stable NP dispersions that show high zeta potential 
values and low agglomeration status, (3) avoid the use of solutions with high ionic strengths, (4) smaller size NPs $(<30 \mathrm{~nm})$ have higher oxidation activity; however, this can change with the aggregation status in the measurement environment, (5) high NP concentrations provide higher signals; however, the concentration of the particles needs to be optimized to prevent light scattering and precipitation. This study demonstrates that nanoceria provides significant advantages in terms of sensitivity and stability, versus the enzyme assay, but the assay requires careful selection of the particles and reaction conditions, in particular ionic composition, particle concentration, surface charge and agglomeration status in the measurement environment.

\section{Acknowledgements}

The authors gratefully acknowledge a David A. Walsh Fellowship to JC. This material is based upon work supported by the National Science Foundation under Grant No. 0954919. Any opinions, findings, and conclusions or recommendations expressed in this material are those of the author(s) and do not necessarily reflect the views of the National Science Foundation. 


\section{References}

[1] Wei $\mathrm{H}$, Wang E. Fe3O4 magnetic nanoparticles as peroxidase mimetics and their applications in H2O2 and glucose detection. Anal Chem. 2008;80:2250-4.

[2] Mu JS, Wang Y, Zhao M, Zhang L. Intrinsic peroxidase-like activity and catalase-like activity of Co3O4 nanoparticles. Chem Commun. 2012;48:2540-2.

[3] Chaudhari KN, Chaudhari NK, Yu J-S. Peroxidase mimic activity of hematite iron oxides ([small alpha]-Fe2O3) with different nanostructures. Catalysis Science \& Technology. 2012;2:119-24.

[4] Fiammengo R, Jaschke A. Nucleic acid enzymes. Curr Opin Biotech. 2005;16:614-21.

[5] Volz $\mathrm{H}$, Holzbecher M. A bridged porphyrinato(thiolato)iron(III) complex as a model of the active center of the cytochrome P-450 isozyme. Angew Chem Int Edit. 1997;36:1442-5.

[6] Ghosh M, Conroy JL, Seto CT. Hydrolysis of amides catalyzed by 4-heterocyclohexanones: Small molecule mimics of serine proteases. Angewandte Chemie-International Edition. 1999;38:514-6. [7] Funabiki T, Yamazaki T, Fukui A, Tanaka T, Yoshida S. Oxygenative cleavage of chlorocatechols with molecular oxygen catalyzed by non-heme iron(III) complexes and its relevance to chlorocatechol dioxygenases. Angewandte Chemie-International Edition. 1998;37:513-5.

[8] Molenveld P, Engbersen JFJ, Reinhoudt DN. Specific RNA dinucleotide cleavage by a synthetic calix[4]arene-based trinuclear metallo(II)-phosphodiesterase. Angewandte Chemie-International Edition. 1999;38:3189-92.

[9] Han MJ, Yoo KS, Chang JY, Ha TK. 5-(beta-cyclodextrinylamino)-5-deoxy-alpha-D-riboses as models for nuclease, ligase, phosphatase, and phosphorylase. Angewandte Chemie-International Edition. 2000;39:347-+.

[10] Muche MS, Gobel MW. Bis(guanidinium) alcohols as models of Staphylococcal nuclease: Substrate binding through ion pair complexes and fast phosphoryl transfer reactions. Angew Chem Int Edit. 1996;35:2126-9.

[11] Signor L, Knuppe C, Hug R, Schweizer B, Pfaltz A, Jaun B. Methane formation by reaction of a methyl thioether with a photo-excited nickel thiolate - A process mimicking methanogenesis in archaea. Chem-Eur J. 2000;6:3508-16.

[12] Wei $\mathrm{H}$, Wang EK. Nanomaterials with enzyme-like characteristics (nanozymes): next-generation artificial enzymes. Chem Soc Rev. 2013;42:6060-93.

[13] Wanga Z, X.; Y, Yanga J, Jianga Y, Heb N. Peroxidase-like activity of mesoporous silica encapsulated Pt nanoparticle and its application in colorimetric immunoassay. Analytica Chimica Acta. 2015; in press.

[14] Wang Cl, Chen WT, Chang HT. Enzyme Mimics of Au/Ag Nanoparticles for Fluorescent Detection of Acetylcholine. Anal Chem. 2012;84:9706-12.

[15] Tang ZW, Wu H, Zhang YY, Li ZH, Lin YH. Enzyme-Mimic Activity of Ferric Nano-Core Residing in Ferritin and Its Biosensing Applications. Anal Chem. 2011;83:8611-6.

[16] Lin YH, Ren JS, Qu XG. Nano-Gold as Artificial Enzymes: Hidden Talents. Adv Mater. 2014;26:4200-17.

[17] Gao LZ, Zhuang J, Nie L, Zhang JB, Zhang Y, Gu N, et al. Intrinsic peroxidase-like activity of ferromagnetic nanoparticles. Nat Nanotechnol. 2007;2:577-83.

[18] Mu JS, Zhang L, Zhao GY, Wang Y. The crystal plane effect on the peroxidase-like catalytic properties of Co3O4 nanomaterials. Phys Chem Chem Phys. 2014;16:15709-16.

[19] Asati A, Santra S, Kaittanis C, Nath S, Perez JM. Oxidase-Like Activity of Polymer-Coated Cerium Oxide Nanoparticles. Angewandte Chemie-International Edition. 2009;48:2308-12.

[20] Chen XM, Tian XT, Su BY, Huang ZY, Chen X, Oyama M. Au nanoparticles on citrate-functionalized graphene nanosheets with a high peroxidase-like performance. Dalton T. 2014;43:7449-54. 
[21] Nath S, Kaittanis C, Ramachandran V, Dalal NS, Perez JM. Synthesis, Magnetic Characterization, and Sensing Applications of Novel Dextran-Coated Iron Oxide Nanorods. Chem Mater. 2009;21:17617.

[22] Tembe S, Karve M, Inamdar S, Haram S, Melo J, D'Souza SF. Development of electrochemical biosensor based on tyrosinase immobilized in composite biopolymeric film. Anal Biochem.

2006;349:72-7.

[23] Tembe S, Inamdar S, Haram S, Karve M, D'Souza SF. Electrochemical biosensor for catechol using agarose-guar gum entrapped tyrosinase. J Biotechnol. 2007;128:80-5.

[24] Alkasir RSJ, Ganesana M, Won YH, Stanciu L, Andreescu S. Enzyme functionalized nanoparticles for electrochemical biosensors: A comparative study with applications for the detection of bisphenol A. Biosens Bioelectron. 2010;26:43-9.

[25] Andreescu S, Sadik OA. Correlation of analyte structures with biosensor responses using the detection of phenolic estrogens as a model. Anal Chem. 2004;76:552-60.

[26] Pirmohamed T, Dowding JM, Singh S, Wasserman B, Heckert E, Karakoti AS, et al. Nanoceria exhibit redox state-dependent catalase mimetic activity. Chem Commun. 2010;46:2736-8.

[27] Andreescu D, Bulbul G, Ozel RE, Sardesai N, Hayat A, Andreescu S. Applications and Implications of Nanoceria Reactivity: Measurement Tools and Environmental Impact Environemtnal Science:

Nano. 2014.

[28] Estevez AY, Erlichman JS. The potential of cerium oxide nanoparticles (nanoceria) for neurodegenerative disease therapy. Nanomedicine. 2014;9:1437-40.

[29] Estevez AY, Pritchard S, Harper K, Aston JW, Lynch A, Lucky JJ, et al. Neuroprotective mechanisms of cerium oxide nanoparticles in a mouse hippocampal brain slice model of ischemia. Free Radical Biology and Medicine. 2011;51:1155-63.

[30] Heckman KL, DeCoteau W, Estevez A, Reed KJ, Costanzo W, Sanford D, et al. Custom Cerium Oxide Nanoparticles Protect against a Free Radical Mediated Autoimmune Degenerative Disease in the Brain. ACS Nano. 2013;7:10582-96.

[31] Li Y, He X, Yin J-J, Ma Y, Zhang P, Li J, et al. Acquired Superoxide-Scavenging Ability of Ceria Nanoparticles. Angewandte Chemie International Edition. 2015;54:1832-5.

[32] Cai X, Sezate SA, Seal S, McGinnis JF. Sustained protection against photoreceptor degeneration in tubby mice by intravitreal injection of nanoceria. Biomaterials. 2012;33:8771-81.

[33] Chigurupati S, Mughal MR, Okun E, Das S, Kumar A, McCaffery M, et al. Effects of cerium oxide nanoparticles on the growth of keratinocytes, fibroblasts and vascular endothelial cells in cutaneous wound healing. Biomaterials. 2013;34:2194-201.

[34] Ornatska M, Sharpe E, Andreescu D, Andreescu S. Paper Bioassay Based on Ceria Nanoparticles as Colorimetric Probes. Anal Chem. 2011;83:4273-80.

[35] Ozel RE, Ispas C, Ganesana M, Leiter JC, Andreescu S. Glutamate oxidase biosensor based on mixed ceria and titania nanoparticles for the detection of glutamate in hypoxic environments.

Biosens Bioelectron. 2014;52:397-402.

[36] Hayat A, Andreescu D, Bulbul G, Andreescu S. Redox reactivity of cerium oxide nanoparticles against dopamine. J Colloid Interf Sci. 2014;418:240-5.

[37] Peng XG, Manna L, Yang WD, Wickham J, Scher E, Kadavanich A, et al. Shape control of CdSe nanocrystals. Nature. 2000;404:59-61.

[38] Fang BZ, Kim MS, Kim JH, Song MY, Wang YJ, Wang HJ, et al. High Pt loading on functionalized multiwall carbon nanotubes as a highly efficient cathode electrocatalyst for proton exchange membrane fuel cells. J Mater Chem. 2011;21:8066-73.

[39] Xue Y, Zhai YW, Zhou KB, Wang L, Tan HN, Luan QF, et al. The Vital Role of Buffer Anions in the Antioxidant Activity of CeO2 Nanoparticles. Chem-Eur J. 2012;18:11115-22.

[40] Singh S, Dosani T, Karakoti AS, Kumar A, Seal S, Self WT. A phosphate-dependent shift in redox state of cerium oxide nanoparticles and its effects on catalytic properties. Biomaterials.

2011;32:6745-53. 
[41] Apetrei C, Rodríguez-Méndez ML, De Saja JA. Amperometric tyrosinase based biosensor using an electropolymerized phosphate-doped polypyrrole film as an immobilization support. Application for detection of phenolic compounds. Electrochimica Acta. 2011;56:8919-25.

[42] Şenyurt Ö, Eyidoğan F, Yılmaz R, Öz MT, Özalp VC, Arıca Y, et al. Development of a paper-type tyrosinase biosensor for detection of phenolic compounds. Biotechnology and Applied Biochemistry. 2014:n/a-n/a.

[43] Qu Y, Ma M, Wang Z, Zhan G, Li B, Wang X, et al. Sensitive amperometric biosensor for phenolic compounds based on graphene-silk peptide/tyrosinase composite nanointerface. Biosensors and Bioelectronics. 2013;44:85-8.

Figure 1. Interaction of seven types of nanoceria particles with $1 \mathrm{mM}$ dopamine in water for the following commercially available particles: a) Cerium (IV) oxide nano particles, 10 wt.dispersion in $\mathrm{H}_{2} \mathrm{O}$ - Aldrich; b) Cerium (IV) oxide dispersion 20 wt. \% colloidal dispersion in $2.5 \%$ acetic acid - Aldrich; c) Cerium oxide, $20 \%$ in $\mathrm{H}_{2} 0$, colloidal dispersion Alfa Aesar; d) Cerium (IV) oxide - 15 - Samaria-doped, nanopowder - Aldrich; e) Cerium (IV) oxide nanopowder (BET) Aldrich; f) Cerium (IV) oxide - 20 - Gadolinium doped - Sigma, nanopowder; g) $\mathrm{CeO}_{2}$ powder Sky Spring; h) control containing only dopamine in water.

Figure 2. Effect of buffer ionic strength on nanoceria interaction with $0.8 \mathrm{mM}$ dopamine. A:; a) $0.05 \mathrm{M}$; b) $0.025 \mathrm{M}$; c) $0.0125 \mathrm{M}$; d) $0.00625 \mathrm{M}$. B: UV-Vis spectra obtained with nanoceria $\left(1 \times 10^{3} \mathrm{mg} / \mathrm{L}\right)$ and dopamine $(0.8 \mathrm{mM})$ in: a) PBS $(0.00625 \mathrm{M}, \mathrm{pH} 8.0)$; b) de ionized water.

Figure 3. Absorption spectra with different concentrations of nanoceria in the presence of 1 $\mathrm{mM}$ dopamine (A) and $0.35 \mathrm{mM}$ catechol (B) for a) 0.125, b) 0.25 , c) 0.5 , d) 0.75 , e) 1 $\left(\mathrm{x} 10^{3} \mathrm{mg} / \mathrm{L}\right)$ particles.

Figure 4. Non-linear fits to Michaelis Menten data for dopamine (a) and catechol (b). 
Figure 5. Calibration curves of the nanoceria assay for a) dopamine and b) catechol (error bars correspond to $\mathrm{n}=3$ independent experiments.

Figure 6.Absorption spectra of of $1000 \mathrm{ppm}$ nanoceria in the presence of (A) a) $0.35 \mathrm{mM}$ catechol and $0.5 \mathrm{mM} \mathrm{b}$ ) dopamine c) glucose d) glycine e) histidinef) $\mathrm{K}$ g) L-glutamic acid h) $\mathrm{Na}$ i) phenylalanine (B). a) $0.25 \mathrm{mM}$ gallic acid b) $0.25 \mathrm{mM}$ quercetin c) $1 \mathrm{mM}$ hydroquinone d) $0.25 \mathrm{mM}$ bisphenol $\mathrm{A}$ e) $1 \mathrm{mM}$ phenol. 


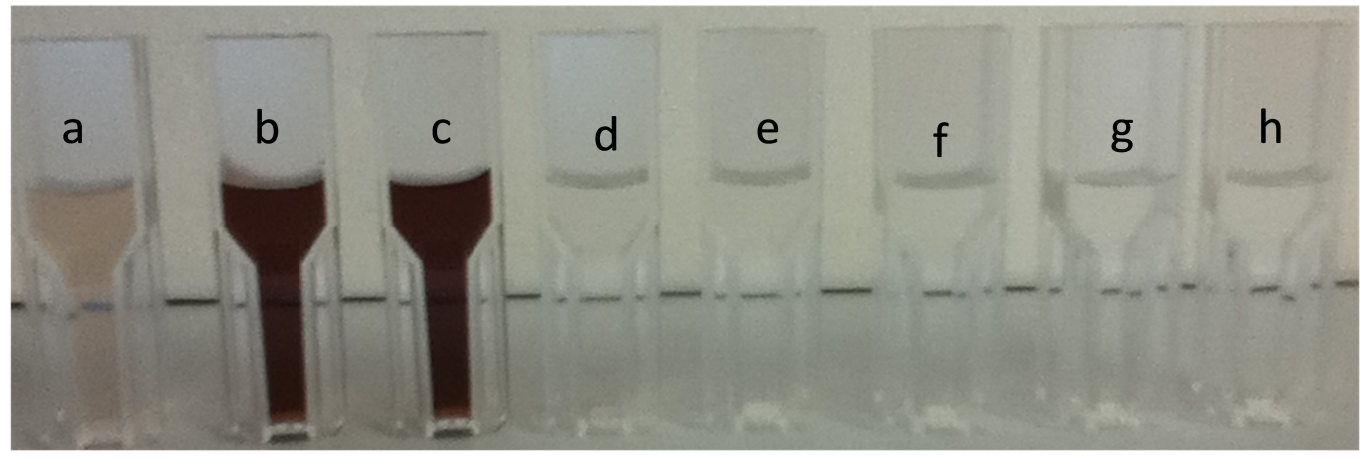

Figure 1 

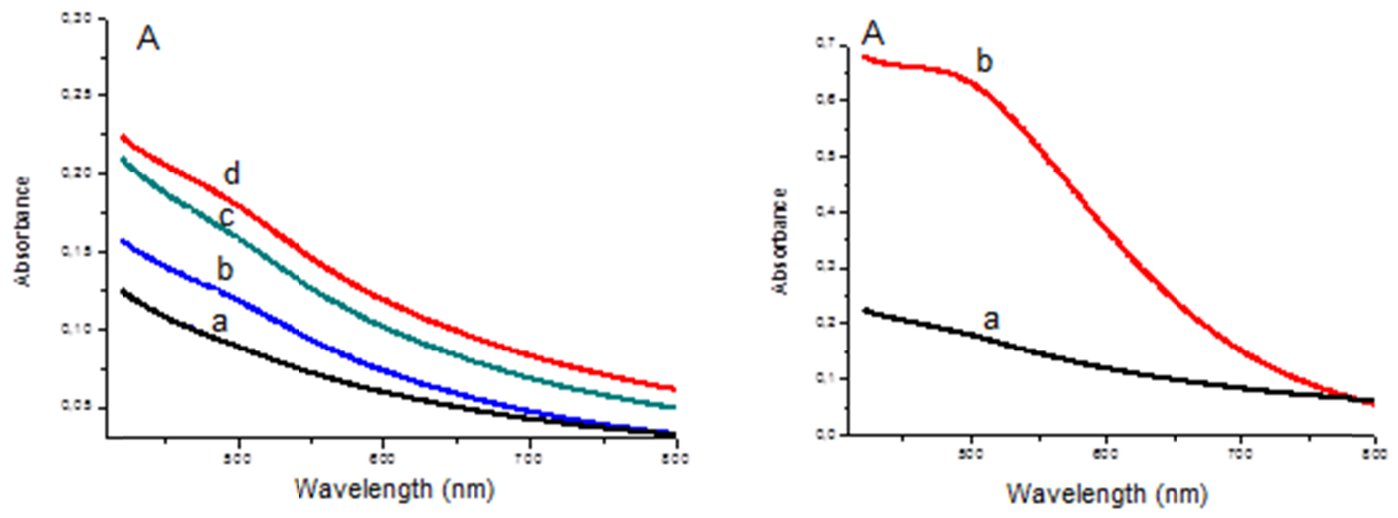

Figure 2 

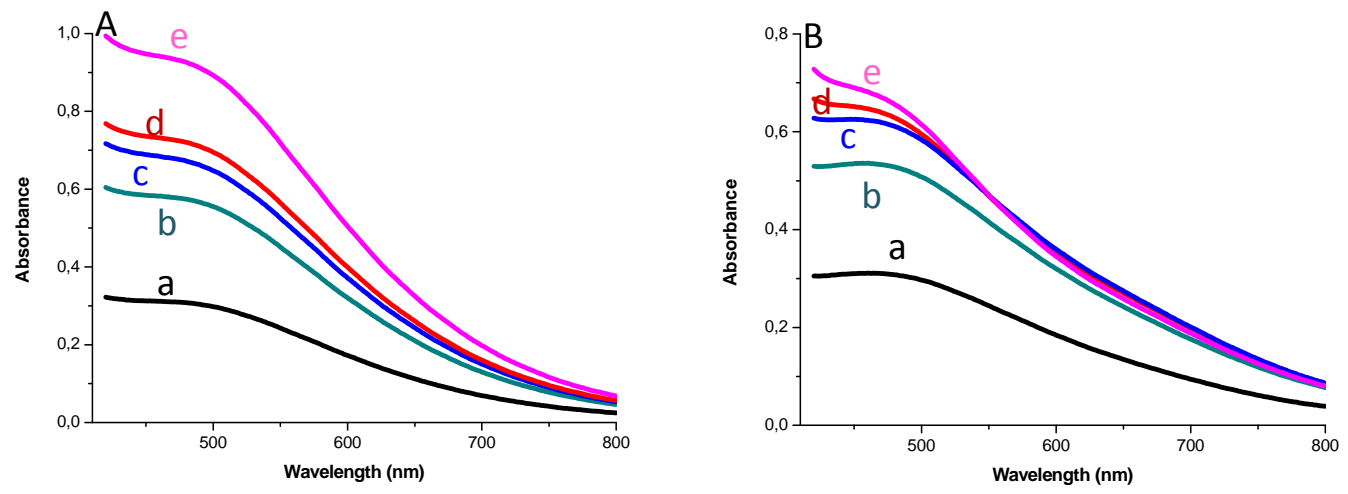

Figure 3 

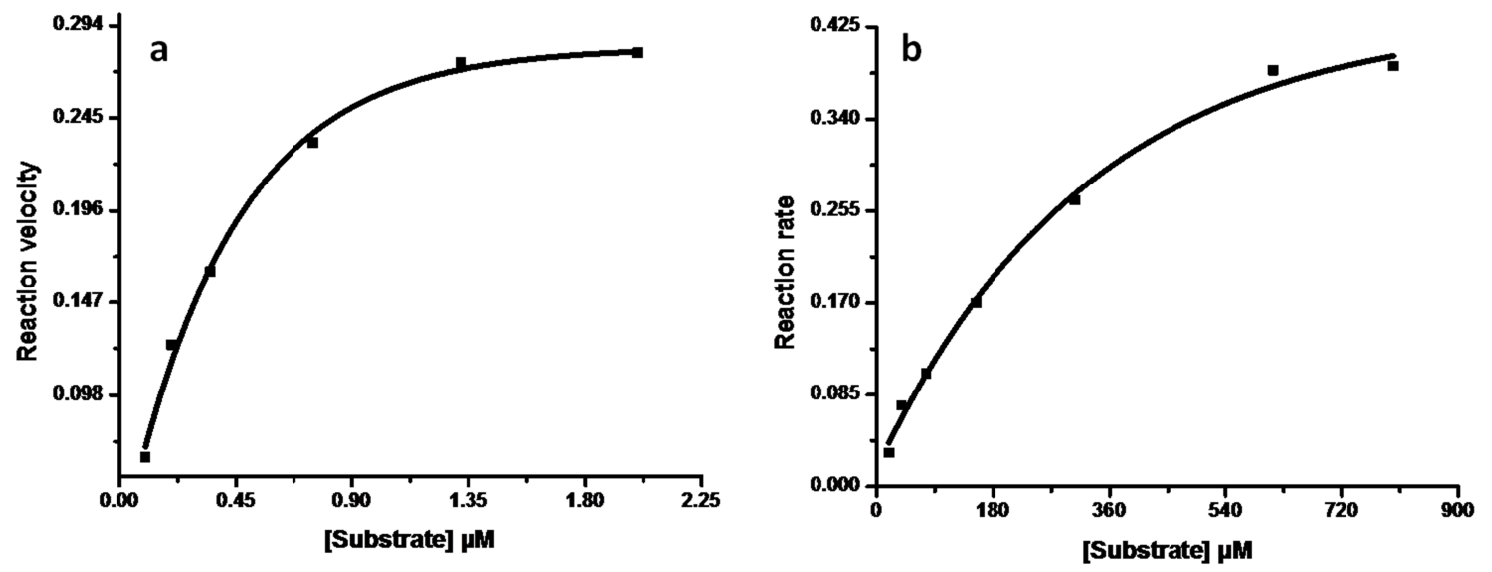

Figure 4 


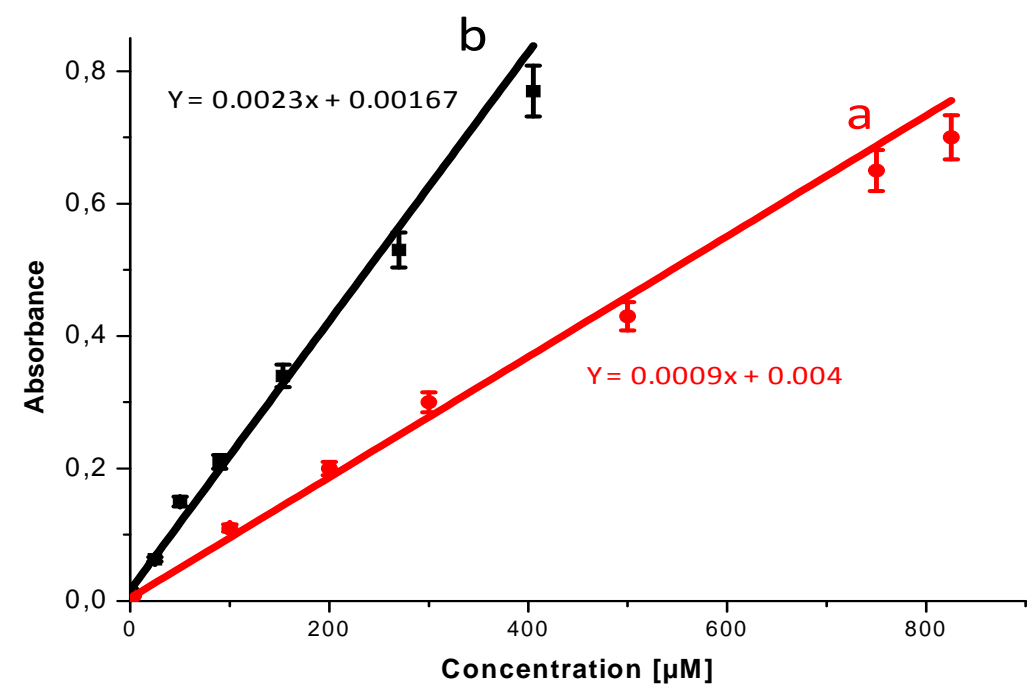

Figure 5 

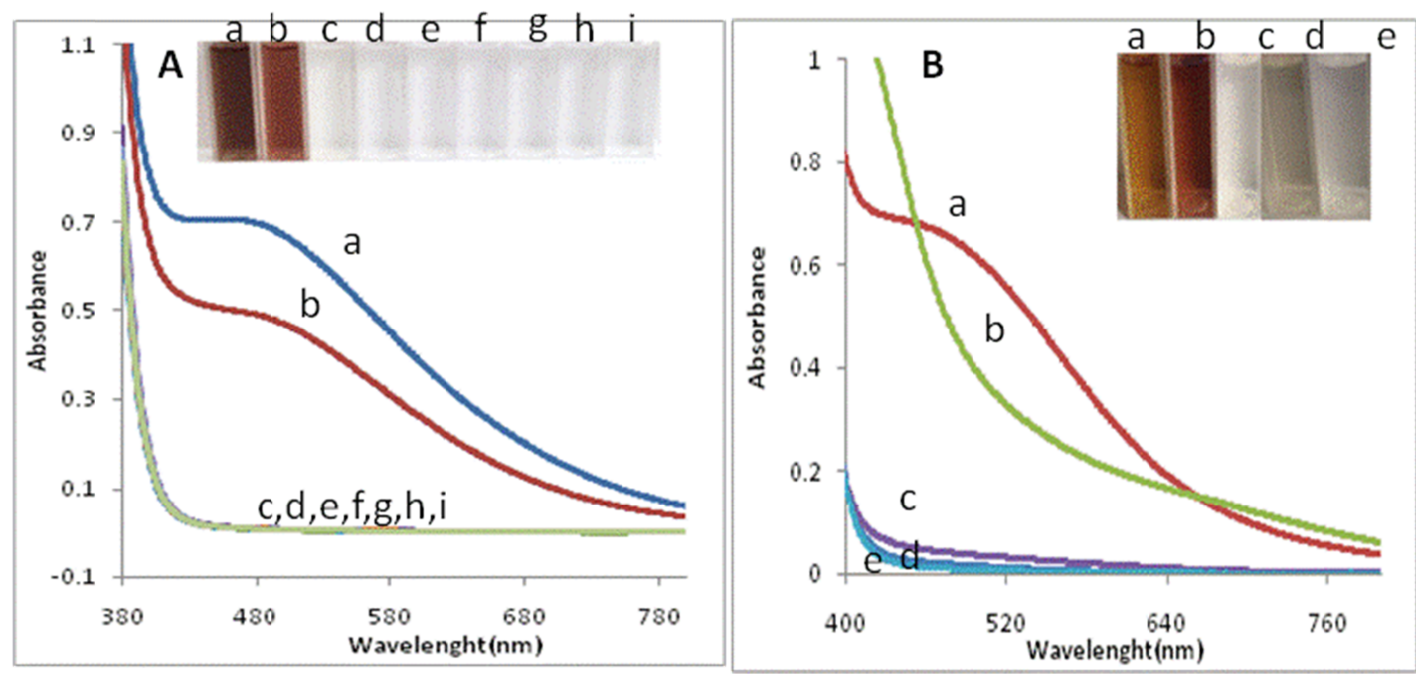

Figure 6. 
Tables

Table 1. Recovery percentages using the nanoceria assay for the analysis of dopamine in spiked human serum and catechol in tape water.

\begin{tabular}{|c|c|c|c|c|c|c|c|}
\hline \multicolumn{5}{|c|}{ Dopamine } & \multicolumn{5}{c|}{ Catechol } \\
\hline Spiked & Determined & ${ }^{\mathrm{a}}$ R.E & ${ }^{\mathrm{b}} \mathrm{R} \%$ & Spiked $(\mu \mathrm{M})$ & Determined & ${ }^{\mathrm{a}} \mathrm{R} . \mathrm{E}$ & ${ }^{\mathrm{b}} \mathrm{R} \%$ \\
& $(\mu \mathrm{M})$ & $\%$ & & & & \\
& & & & & & \\
\hline 250 & 240 & 4 & 96 & 100 & 95 & 5 & 95 \\
\hline 400 & 392 & 2 & 98 & 200 & 187 & 6.5 & 93.5 \\
\hline 500 & 508 & 1.6 & 101.6 & 350 & 335 & 4.29 & 95.71 \\
\hline
\end{tabular}

${ }^{a}$ relative $\%$ error $;{ }^{b} \%$ recovery

Table 2. Analytical performance characteristics of the nanoceria assays as compared to the tyrosinase based detection of dopamine and catechol using optimized nanoceria and tyrosinase concentrations.

\begin{tabular}{|c|c|c|c|c|}
\hline & \multicolumn{2}{|c|}{ Dopamine } & \multicolumn{2}{|c|}{ Catechol } \\
\hline & Tyrosinase & Nanoceria & Tyrosinase & Nanoceria \\
\hline${\text { Sensitivity }(\mu \mathrm{M})^{-1}}^{-1}$ & 0.00029 & 0.0009 & 0.00076 & 0.0023 \\
\hline LOD $(\mu \mathrm{M})$ & 50 & 1.5 & 30 & 0.2 \\
\hline SD & \pm 2.5 & \pm 0.075 & \pm 1.5 & \pm 0.01 \\
\hline $\mathbf{R}^{2}$ & 0.99 & 0.99 & 0.97 & 0.983 \\
\hline
\end{tabular}




\section{Graphical abstract}
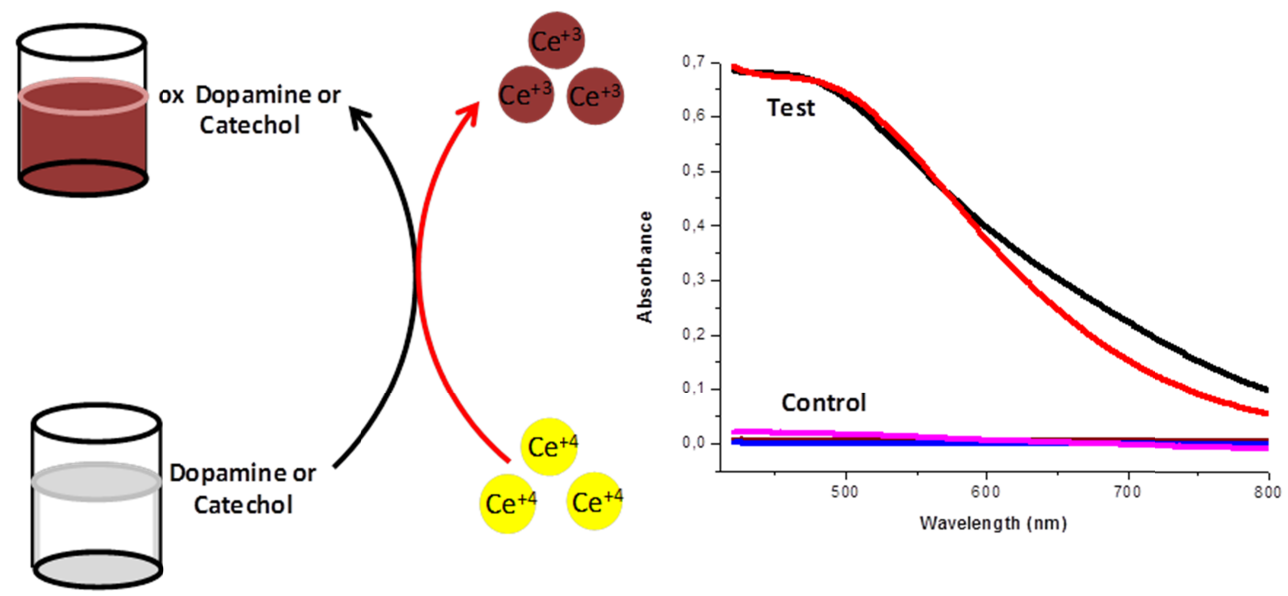